\title{
Outcomes of the association
} of asymptomatic cystocele repair in patients undergoing mid-urethral sling surgery for the correction of stress incontinence

\author{
Ahmed Yehia Abdel Aziz ${ }^{1 *}$, Sarah Hassan², Samer Morsy ${ }^{1}$, Hussein Aly Hussein ${ }^{1}$, Dalia Abdel Azim² \\ and Mohamed Salah Abdel Azim ${ }^{1}$
}

\begin{abstract}
Background: Comparison between the clinical outcomes of mid-urethral sling procedure for stress incontinence with and without repair of asymptomatic stage II cystocele is made.

Methods: This is a prospective randomized study of 72 female patients with stress urinary incontinence and asymptomatic stage II cystocele. The patients were divided into two groups: group 1 treated with trans-obturator tape only and group 2 with repair of cystocele by anterior colporrhaphy in the same session. We compared between both groups in cure rate and voiding function after 1 year.

Results: The cure rate of group 1 was $66.7 \%$, while for group 2 it was $90 \%$ after 12 months $(p<0.05)$. Six patients (17\%) with asymptomatic stage II cystocele in group 1 became symptomatic or developed higher stage after 12 months that required surgical repair. The incidence of postoperative irritative urinary symptoms was also significantly higher in group 1. There were no significant differences in the change in maximum flow rate or postvoiding residual urine between the two groups postoperatively.
\end{abstract}

Conclusion: Concomitant repair of stage II asymptomatic cystocele with mid-urethral sling improves the cure rate of stress urinary insentience and reduces the incidence of irritative urinary symptoms.

Keywords: Cystocele, Mid-urethral sling, Stress urinary incontinence, Trans-obturator tape

\section{Background}

International Continence Society defines stress urinary incontinence (SUI) as involuntary leakage of urine with effort or any exertion, or with sneezing or coughing [1]. The stress urinary incontinence may be due to urethral hypermobility or intrinsic sphincter deficiency (ISD); however, both conditions can coexist [2].

Eleven percent of women by the age of 80 years needs surgical repair for SUI; $29.2 \%$ of these patients need more

\footnotetext{
*Correspondence: dr_ahmedyehia81@yahoo.com

1 Urology Department, Faculty of Medicine, Cairo University, Cairo 11562, Egypt

Full list of author information is available at the end of the article
}

than one surgery [3]. Mid-urethral sling is currently the most common effective procedure that is performed to correct SUI.

Cases that undergo surgical intervention for SUI have a high incidence of associated anterior vaginal prolapse that requires surgical repair $[4,5]$. Pelvic organ prolapse is defined as the abnormal descent of anterior or posterior vaginal wall or apex of the vagina or vaginal vault after hysterectomy $[6,7]$.

The correction of asymptomatic stage II cystocele is still debatable. The study aimed to assess the value of concomitant surgical correction of asymptomatic stage 
II anterior vaginal wall prolapse with treatment of female patients with stress incontinence by mid-urethral sling.

\section{Methods}

A prospective study of 72 female patients presented to the Urology Department, with stress urinary incontinence (SUI) and associated asymptomatic stage II cystocele in the period between June 2014 and April 2016.

This is a prospective randomized study where patients were randomly divided into two groups by simple randomization using computer generated random numbers by Microsoft Excel. Group 1 was treated only with transobturator tape (TOT), and group 2 was treated with TOT and concomitant repair of cystocele by anterior colporrhaphy.

The study was performed according to the ethics principles of the 1975 Declaration of Helsinki. A written informed consent was obtained from all patients. The study protocol and the suggested informed consent were approved by the Institutional Review Board (IRB) of the Urology Department before the start of the study.

We included in the study patients with clinical SUI by patients' complaint and positive stress test associated with asymptomatic stage II anterior compartment vaginal wall prolapse (cystocele).

We excluded patients with symptomatic cystocele, past history of mid-urethral sling or cystocele repair, other compartment prolapse or with any neurological disease. The stage of cystocele was identified according to the Pelvic Organ Prolapse Quantification (POP-Q) system. POP-Q system defines stage II cystocele as the condition that The most distal prolapse is between $1 \mathrm{~cm}$ above and $1 \mathrm{~cm}$ below the hymen (at least one point is $-1,0$ or +1 ).

Preoperative evaluation for all patients included clinical history of urinary symptoms using American Urological Association Symptom Score (AUASS) questionnaire, abdominal and vaginal examination, urine analysis and culture, and pelvic ultrasound to measure postvoiding residual urine.

Mid-urethral slings were fixed in all patients to correct stress urinary incontinence. Trans-obturator tape (Dynamesh-SIS soft tape) was used in all cases using inside-out technique. We repaired the prolapse by classic anterior colporrhaphy without using synthetic mesh to avoid its complications. Cystoscopy was done at the end of the procedure to exclude bladder injury during the procedure. All procedures were done by the same team. Vaginal pack and urethral catheter were removed on the morning of the next day.

We compared between both groups regarding postoperative clinical improvement in the stress urinary incontinence, urinary symptoms using AUASS questionnaire, development of de novo symptoms, postoperative Q-max urine flow and postvoiding residual urine by ultrasound. Clinical cure of stress urinary incontinence was detected simply by asking the patients about the persistence of stress urinary incontinence, and we also used pad test to confirm this improvement, and provocative stress test.

Anatomical failure of the procedure was diagnosed if the cystocele was still stage II or higher, and anatomical success was defined if cystocele became stage 0 or I. Intraoperative and postoperative complications were carefully evaluated.

\subsection{Statistical analysis of data}

Comparison between categorical data in different studied groups was made using Chi-square test. Comparison between mean values of $\mathrm{Q}$ in the studied groups was made using $T$ test. Statistical Package for Social Sciences (SPSS) computer program (version 19 windows) was used for data analysis. $p$ value $\leq 0.05$ was considered significant and $<0.01$ was considered highly significant.

\section{Results}

Between June 2014 and April 2016, 84 women presented to our department with SUI and asymptomatic stage II cystocele. Twelve patients refused to follow our study protocol. Our study involved 72 patients (36 patients treated with TOT only and 36 patients with concomitant repair of cystocele).

Preoperative complete history examinations were done to all patients. Preoperative demographic data of the two groups were comparable with no significant difference. There were no significant differences between the two groups in age, preoperative body mass index (BMI) and vaginal deliveries (Table 1 ).

The incidence of urgency urinary incontinence was more in concomitant repair group, but with no significant difference. There was no significant difference between both groups regarding irritative and obstructive urinary symptoms, maximum urine flow rate $(\mathrm{Q} \max )$ and residual urine. The total AUASS was 18.3 in the TOT only group and 16.5 in the concomitant repair group ( $p$ value 0.43 ) (Table 2 ).

Table 1 Demographic data

\begin{tabular}{llll}
\hline & TOT only group & $\begin{array}{l}\text { Concomitant } \\
\text { repair group }\end{array}$ & p value \\
\hline Number of patients & 36 & 36 & - \\
Age (years) & $49.7 \pm 8.5$ & $52 \pm 9.4$ & 0.264 \\
BMl $\left(\mathrm{kg} / \mathrm{m}^{2}\right)$ & $28.87 \pm 2.9$ & $30 \pm 3.1$ & 0.687 \\
Vaginal deliveries & $2.40 \pm 1.5$ & $2.1 \pm 1.4$ & 0.538 \\
\hline
\end{tabular}

TOT Trans-obturator tape, BMI body mass index 
Table 2 Preoperative urinary symptoms

\begin{tabular}{llll}
\hline & TOT only group & $\begin{array}{l}\text { Concomitant } \\
\text { repair group }\end{array}$ & $\boldsymbol{p}$ value \\
\hline Qmax $(\mathrm{ml} / \mathrm{s})$ & $26.4 \pm 9.6$ & $23.5 \pm 10.2$ & 0.356 \\
PVR $(\mathrm{ml})$ & $35.7 \pm 20.2$ & $40.1 \pm 24.4$ & 0.231 \\
AUASS & $18.3 \pm 9.1$ & $17.5 \pm 8.4$ & 0.425 \\
Frequency & $15(41.7 \%)$ & $18(50 \%)$ & 0.478 \\
Urgency & $18(50 \%)$ & $24(66.7 \%)$ & 0.151 \\
Urgency incontinence & $16(44.4 \%)$ & $21(58.3 \%)$ & 0.238 \\
Weak flow & $4(11.1 \%)$ & $3(8.3 \%)$ & 1 \\
Interrupted flow & $4(11.1 \%)$ & $2(5.6 \%)$ & 0.674 \\
\hline
\end{tabular}

TOT trans-obturator tape, $Q$ max Maximum flow rate, $P V R$ postvoiding residual urine, AUASS American Urological Association Symptom Score

Table 3 Postoperative urinary symptoms

\begin{tabular}{llll}
\hline & TOT only group & $\begin{array}{l}\text { Concomitant } \\
\text { repair group }\end{array}$ & $p$ value \\
\hline Cure rate & $66.7 \%$ & $90 \%$ & $0.002^{*}$ \\
Change in Q max (ml/s) & $-4.7 \pm 14.0$ & $-3.8 \pm 9.4$ & 0.8 \\
Change in PVR (ml) & $10 \pm 32.9$ & $20 \pm 60.2$ & 0.3 \\
Postoperative AUASS & $20.5 \pm 8.2$ & $6.2 \pm 5.3$ & $0.01^{*}$ \\
Frequency & $20(55.5 \%)$ & $8(22.2 \%)$ & $0.004^{*}$ \\
Urgency & $27(75 \%)$ & $10(27.7 \%)$ & $<0.001^{*}$ \\
Urgency incontinence & $25(69.4 \%)$ & $8(22.2 \%)$ & $<0.001^{*}$ \\
Weak flow & $2(5.6 \%)$ & $2(5.6 \%)$ & 1 \\
Interrupted flow & $2(5.6 \%)$ & $1(2.7 \%)$ & 1 \\
\hline
\end{tabular}

TOT trans-obturator tape, Q max maximum flow rate, $P V R$ postvoiding residual urine, AUASS American Urological Association Symptom Score

Follow-up of all patients was postoperatively at 1 weak, 1, 3, 6 months and 1 year. We compared the postoperative results between both groups at 12-month follow-up to detect incidence of cure rate, progression of irritative or obstructive symptoms, de novo symptoms and the need for other interventions.
The postoperative AUASS were significantly higher in the TOT only group $(20.5 \pm 8.2$ in comparison with $6.2 \pm 5.3$ in concomitant repair group). The incidence of postoperative irritative symptoms (frequency, urgency and urgency incontinence) was also significantly higher in TOT only group. There were no significant differences in the change in urine maximum flow or PVR between the two groups postoperatively. The incidence of postoperative obstructive symptoms was very low with no difference between both groups (Table 3).

Table 4 shows the postoperative outcome in comparison with preoperative findings in both groups. There was significant postoperative improvement in irritative urinary symptoms (frequency, urgency and urgency incontinence) and AUASS in concomitant repair group, while there was no significant difference in the other group. There were no postoperative differences in comparison with preoperative findings in both groups regarding obstructive urinary symptoms, $\mathrm{Q} \max$ and PVR.

For clinical outcomes based on patient's subjective symptoms, pad test and stress test, the cure rate of TOT only group was $66.7 \%$ at 12 months; meanwhile, the cure rate of concomitant repair group was $90 \%$, ( $p$ value 0.002 ) (Table 3). Cystocele was cured in all patients in concomitant repair group; six patients (17\%) with asymptomatic cystocele in TOT only group became symptomatic or developed a higher stage at 12 months duration that required surgical repair.

There was significant improvement in the sexual activity in all patients postoperatively as there was significant decrease in the coital incontinence. There were no intraoperative significant complications in all patients; follow-up for 12 months showed no postoperative significant TOT complications.

Table 4 Postoperative outcome in each group

\begin{tabular}{|c|c|c|c|c|c|c|}
\hline & Preoperative TOT only & $\begin{array}{l}\text { Postoperative } \\
\text { TOT only }\end{array}$ & $p$ value & $\begin{array}{l}\text { Preoperative } \\
\text { concomitant repair }\end{array}$ & $\begin{array}{l}\text { Postoperative } \\
\text { concomitant repair }\end{array}$ & $p$ value \\
\hline Frequency & 15 & 20 & 0.238 & 18 & 8 & $0.014^{*}$ \\
\hline Urgency & 18 & 27 & $0.028^{*}$ & 24 & 10 & $0.001^{*}$ \\
\hline Urgency incontinence & $16(44.4 \%)$ & 25 & $0.032^{*}$ & $21(58.3 \%)$ & 8 & $0.002^{*}$ \\
\hline Weak flow & 4 & 2 & 0.674 & 3 & 2 & 1 \\
\hline Interrupted flow & 4 & 2 & 0.674 & 2 & 1 & 1 \\
\hline AUASS & $18.3 \pm 9.1$ & $20.5 \pm 8.2$ & 0.357 & $17.5 \pm 8.4$ & $6.2 \pm 5.3$ & $0.02^{*}$ \\
\hline Qmax (ml/s) & $26.4 \pm 9.6$ & $21.7 \pm 10.4$ & 0.273 & $23.5 \pm 10.2$ & $19.7 \pm 11.2$ & 0.264 \\
\hline PVR (ml) & $35.7 \pm 20.2$ & $45 \pm 21.5$ & 0.145 & $40.1 \pm 24.4$ & $60 \pm 26.4$ & 0.154 \\
\hline
\end{tabular}

TOT trans-obturator tape, Q max Maximum flow rate, PVR Postvoiding residual urine, AUASS American Urological Association Symptom Score 


\section{Discussion}

Urinary incontinence and anterior vaginal wall prolapse occur simultaneously in aging women. We studied the value of concomitant repair of asymptomatic stage II cystocele with mid-urethral sling for SUI. We found significant higher cure rate of SUI in patients with concomitant repair of cystocele with less irritative urinary symptoms and no obstructive symptoms.

Many studies found that sever cystocele may affect the results of SUI treatment with mid-urethral sling. Paick et al. found that a severe grade of cystocele is associated with persistent SUI after sling surgical repair. They also reported that severe grade of cystocele caused voiding dysfunction when uncorrected after sling surgery, because the urethral angle that induced by sling surgery may be aggravated and become worse by remaining cystocele [8]. Deffieux et al. also reported that the presence of severe anterior vaginal wall prolapse during mid-urethral sling surgery may cause voiding dysfunction and affect surgical outcome if left uncorrected [9]. Reich et al. found that sling without correction of anterior vaginal wall prolapse may aggravate the already existing anterior vaginal wall prolapse and has poor impact on cure rates of sling procedures resulting in sling failure [10].

Most of the studies on concomitant repair focused on patients with severe cystocele, such as stages III and IV. Little is known about the value of concomitant repair of mild stage cystocele. Our study showed that there was a difference in cure rate between TOT only group and the other with concomitant cystocele repair in favor of concomitant repair (66.7\% vs. $90 \%)$. De novo urgency is one of the common complications which may occur after anti-incontinence surgery, and the patients should be informed and consented preoperatively [11]. In our study, higher incidence of frequency and urgency with or without urgency urinary incontinence was found in TOT only group compared to the other group.

Lleberia and coworkers reported in their review of the literature that the incidence of de novo urgency is between 3.1 and $25.9 \%$ after mid-urethral sling and it is with unclear pathophysiology [12]. Nguyen and coworkers reported that surgical correction of the symptomatic prolapse reduces urgency symptoms in $63 \%$ of patients [13].

In our study, the cystocele was repaired by anterior colporrhaphy without the use of any synthetic mesh to avoid unnecessary complications in mild cystocele. Zambon JP et al. reported that the incidence of postoperative vaginal mesh for prolapse remains elevated despite improvement in outcome [14].

Our study showed no postoperative obstructive symptoms. Although the preoperative Qmax was slightly lower in the concomitant repair group, the postoperative change in Qmax was similar between the two groups, and postoperative PVR was not significantly different between the two groups. This is usually because stage II cystocele is small to induce a change in urethral angulation that may cause urinary obstruction.

Park et al. reported different results as they found that stage II cystocele does not affect the cure rate or voiding function after SUI repair with sling surgery, irrespective of cystocele repair. They concluded that the correction of asymptomatic mild degree of cystocele is not mandatory in sling surgery [15].

Anger and coworkers found that the incidence of occurrence of anterior wall vaginal prolapse was found to be higher in the year after the sling operation and that patients who underwent concomitant prolapse surgery with sling procedure were less likely to need prolapse repair later on than those who underwent a sling surgery alone. They also found that the cases treated with sling without the repair of asymptomatic anterior wall vaginal prolapse became symptomatic after the sling procedure and usually require surgical management within 1 year [16].

Many studies show positive influence of mid-urethral sling on sexual function [17]. Our study showed significant improvement in the sexual activity in all patients postoperatively as there was significant decrease in the coital incontinence. Surgical repair of prolapse using synthetic mesh usually has negative effect on sexual function in comparison with anterior colporrhaphy [18]. We did not use synthetic mesh during surgical repair of cystocele to avoid its complications.

Our study suggested that the correction of all pelvic floor dysfunction in the same procedure is proposed to show more superior clinical benefit for the patients and limits their exposure to other procedures that may be depressing for the patients. From surgical point of view, exposure of the patients to one surgical intervention reduces the overall recovery phase and avoids more technical difficulties because of fibrosis and scarring.

Our study has some limitations. First, the 1-year follow-up is considered relatively short and we need more follow-up. Second, there was no preoperative and postoperative urodynamic study to all patients and we used only uroflowmetry and a questionnaire to compare surgical success rate and voiding function.

In spite of that, our study is one of the few studies that analyze the effect of concomitant stage II anterior wall vaginal prolapse repair with TOT on success rate. We found a significant increase in the success rate of stress incontinence in cases with asymptomatic cystocele when it was repaired by anterior colporrhaphy. 


\section{Conclusion}

Concomitant repair of stage II asymptomatic cystocele with mid-urethral sling improves the cure rate of stress urinary insentience and reduces the incidence of irritative urinary symptoms.

\section{Abbreviations}

TOT: trans-obturator tape; SUI: stress urinary incontinence; ISD: intrinsic sphincter deficiency; IRB: Institutional Review Board; POP-Q: pelvic organ prolapse quantification; AUASS: American Urological Association Symptom Score.

\section{Acknowledgements}

None.

\section{Authors' contributions}

$\mathrm{HAH}$ designed the study; SM and SH were involved in the acquisition of data; DA analyzed the data; AA interpreted the data, and drafted and submitted the article; and MSA revised the article and gave final approval of the version. All authors have read and approved the manuscript.

\section{Funding}

There is no source of funding.

\section{Availability of data and materials}

All raw data and materials are available.

\section{Ethics approval and consent to participate}

Written consents were obtained from the participants before the study. The ethical committee and Institutional Review Board (IRB) of the Urology Department, Cairo University, approved this study in January 2014.

\section{Consent for publication}

Not applicable.

\section{Competing interests}

The authors declare that they have no competing interests.

\section{Author details}

${ }^{1}$ Urology Department, Faculty of Medicine, Cairo University, Cairo 11562,

Egypt. ${ }^{2}$ Obstetrics and Gynecology Department, Cairo University, Cairo, Egypt.

Received: 23 February 2020 Accepted: 2 June 2020

Published online: 09 July 2020

\section{References}

1. Abrams P, Cardozo L, Fall M, Griffiths D, Rosier P, Ulmsten U et al (2003) The standardization of terminology in lower urinary tract function: report from the standardization sub-committee of the International Continence Society. Urology 61(1):37-49

2. Nitti VW, Combs AJ (1996) Correlation of Valsalva leak point pressure with subjective degree of stress urinary incontinence in women. J Urol 155:281-285
3. Olsen AL, Smith VJ, Bergstrom JO, Colling JC, Clark AL (1997) Epidemiology of surgically managed pelvic organ prolapse and urinary incontinence. Obstet Gynecol 89(4):501-506

4. Ng CS, Rackley RR, Appell RA (2001) Incidence of concomitant procedures for pelvic organ prolapse and reconstruction in women who undergo surgery for stress urinary incontinence. Urology 57(5):911-913

5. Morgan TO, Westney OL, McGuire EJ (2000) Pubovaginal sling: 4-year outcome analysis and quality of life assessment. J Urol 163(6):1845-1848

6. Hagen S, Thakar R (2012) Conservative management of pelvic organ prolapse. Obstet Gynaecol Reprod Med 22(5):118-122

7. Stanton SL, Cardozo LD (1980) Surgical treatment of incontinence in elderly women. Surg Gynecol Obstet 150(4):555-557

8. Paick JS, Cho MC, Oh SJ, Kim SW, Ku JH (2007) Factors influencing the outcome of mid urethral sling procedures for female urinary incontinence. J Urol 178(3):985-989

9. Deffieux X, Daher N, Mansoor A, Debodinance P, Muhlstein J, Fernandez H (2010) Transobturator TVT-O versus retropubic TVT: results of a multicenter randomized controlled trial at 24 months follow-up. Int Urogynecol J 21(11):1337-1345

10. Reich A, Kohorst F, Kreienberg R, Flock F (2011) Long-term results of the tension-free vaginal tape procedure in an unselected group: a 7-year follow-up study. Urology 78(4):774-777

11. Kwong PO, Westney OL (2005) Management of postoperative detrusor instability and voiding dysfunction. In: Vasavada SP, Appell RA, Sand PK, Raz S (eds) Female urology, urogynecology, and voiding dysfunction. Marcel Dekker, New York, pp 437-446

12. Lleberia J, Pubill J, Mestre M, Aguiló O, Serra L, Canet $Y$ (2013) De novo urgency: a review of the literature. Gynecol Obstet 3:166. https://doi. org/10.4172/2161-0932.1000166

13. Nguyen JK, Bhatia NN (2001) Resolution of motor urge incontinence after surgical repair of pelvic organ prolapse. J Urol 166(6):2263-2266

14. Zambon JP, Badlani GH (2016) Vaginal mesh exposure presentation, evaluation, and management. Curr Urol Rep. 17(9):65. https://doi. org/10.1007/s11934-016-0617-Z

15. Park HK, Paick SH, Lho YS, Choo GY, Kim HG, Choi J (2013) Lack of effect of concomitant stage II cystocele repair on lower urinary tract symptoms and surgical outcome after tension-free vaginal tape procedure: randomized controlled trial. Int Urogynecol J 24(7):1123-1126. https://doi. org/10.1007/s00192-012-1961-2 (Epub 2012 Nov 3)

16. Anger JT, Litwin MS, Wang Q, Pashos CL, Rodríguez LV (2007) Complications of sling surgery among female medicare beneficiaries. Obstet Gynecol 109(3):707-714

17. Mengerink BB, Van Leijsen SA, Vierhout ME, Inthout J, Mol BW, Milani AL et al (2016) The impact of midurethral sling surgery on sexual activity and function in women with stress urinary incontinence. J Sex Med 13(10):1498-1507. https://doi.org/10.1016/j.jsxm.2016.08.005

18. Vollebregt A, Fischer K, Gietelink D, van der Vaart CH (2012) Effects of vaginal prolapse surgery on sexuality in women and men; results from a RCT on repair with and without mesh. J Sex Med 9(4):1200-1211. https:// doi.org/10.1111/j.1743-6109.2011.02647.x (Epub 2012 Feb 9)

\section{Publisher's Note}

Springer Nature remains neutral with regard to jurisdictional claims in published maps and institutional affiliations. 